\title{
Chromosome Analysis Using Spectral Karyotyping (SKY)
}

\author{
George Imataka · Osamu Arisaka
}

Published online: 27 September 2011

(c) The Author(s) 2011. This article is published with open access at Springerlink.com

\begin{abstract}
Spectral karyotyping is a novel technique for chromosome analysis that has been developed based on the approach of the fluorescence in situ hybridization technique. Spectral karyotyping makes it feasible to diagnose a variety of diseases, because of its technology in painting each of the 24 human chromosomes with different colors. In recent years, it has become possible to adopt the usage of spectral karyotyping for research in general clinical practice, and its usability has attracted particular attention in the diagnosis of different diseases. In this review, we will explain the principle of the spectral karyotyping, as well as its specificity and limitation in detecting the genetic defects within clinical application by presenting two case reports.
\end{abstract}

Keywords Spectral karyotyping · Fluorescent in situ hybridization · Chromosome SKY · Multicolor FISH

\section{Introduction}

The first report of human chromosomes was made in 1882 by Flemming, in which $22-28$ chromosomes in the dividing cells of the corneal epithelium were described [1]. Forty years later, Painter reported that the number of human chromosomes was 48 and that sex was determined according to the presence or absence of the $\mathrm{Y}$ chromosome [1]. It was not until the report of " $2 n=46$ " was made in 1956 by Tjio and Levan that the nature of human chromosomes was further established [1]. Following this report,

G. Imataka $(\bowtie) \cdot$ O. Arisaka

Department of Pediatrics, Dokkyo Medical University, 880 Mibu, Shimotsuga, Tochigi 321-0293, Japan

e-mail: geo@dokkyomed.ac.jp the relationships between various congenital disorders and chromosomal abnormalities have been revealed one after another. In 1959, Lejeune et al. [2] demonstrated that Down syndrome is caused by the presence of an extra chromosome 21, i.e., trisomy [3]. This finding of chromosome 21-trisomy was a turning point in the subsequent discovery of several other trisomy syndromes, including chromosomes 18 [4, 5], 13 [6], 8 [7], and 22 [8, 9]. Besides the chromosomal number aberrations, abnormalities associated with sex chromosomes, viz., Turner syndrome [10] and Klinefelter syndrome [11] and other chromosomes $[5,12]$ have been reported.

An increased understanding of malignant diseases in the $1960 \mathrm{~s}$ and the advent of better cell culture methods including a method of culturing peripheral blood [13] led to the first discovery of chromosomal abnormalities in malignant tumors, the $\mathrm{Ph}^{1}$ chromosome, which is specific to chronic myelogenous leukemia [14]. Genetic analysis studies, represented by the Human Genomic Project [15], led to the application of modern genetics tools in research in biological phenomena such as the development, evolution, and aging, as well as abnormal cellular differentiation including cancers.

Therefore, in the course of studying human chromosomes for more than a century, many new techniques have been developed for chromosome testing. In 1971, Caspersson, et al. [16] developed Q-staining using quinacrine mustard, a fluorescent dye that binds to DNA, which was instrumental in the discovery that chromosomes have banding patterns (stripes). Later, improved staining techniques, including $\mathrm{R}-, \mathrm{T}-, \mathrm{C}-$, and $\mathrm{G}$-staining, have appeared. The analysis of the band has further advanced and now it has been developed into high-resolution chromosome banding [17]. Furthermore, along with the development of the fluorescent in situ hybridization (FISH) 
technique [18], which involves the mapping of a chromosome using fluorescent labeled probe, it has become possible to identify chromosomal microdeletion and structural abnormalities such as the extra portion of a chromosome and a marker chromosome. FISH has also been used in diagnosing abnormalities, caused by chromosomal aneuploidy — which are difficult to detect via conventional test methods.

Comparative genomic hybridization (CGH) [19] and spectral karyotyping (SKY) [20], which are based on the FISH technique, are relatively recent and improved techniques of chromosomal analysis. SKY as described here refers to the multicolor-FISH technique, which makes it possible to identify 24 human chromosomes via single hybridization and then to stain them with different colors. In this article, the analysis of chromosomal abnormalities using the SKY technique and its limitation for detection will be discussed.

\section{Principle of SKY}

Two techniques are used as the basic principles of FISH: chromosome painting and multicolor fluorescence. The former is a technique of drawing an entire image of certain chromosome by using fluorescent signals. The latter is a technique of drawing images of several hybridization signals with different florescent dyes. Combining these two techniques, in 1996, the SKY technique was developed by Schrock et al. [20].

In SKY, the color emission of chromosomes is determined by the combination of painting probes and (fluorescent dyes). In this technique, new colors can be developed by extracting a pair of different fluorescent dyes from among the five types of fluorescent dyes, spectrum orange, Texas red, Cy5, spectrum green, Cy5.5, and mixing each pair together. Thus, it is theoretically possible to create $2^{N}-1$ number of colors from $N$ types of fluorescent dyes. Therefore, 31 types of colors can be created from five types of fluorescent dyes. In reality, however, because some fluorescence has a wavelength close to that within the infrared spectrum, a two-dimensional imaging spectroscopy system (e.g., the Spectral Bio-Imaging System SD-200, Applied Spectral Imaging Ltd. Israel) needs to be used to process spectral images so that 24 macroscopically distinguishable colors (Table 1) can be created.

The amount of samples required for the test is $3.0 \mathrm{ml}$ of heparin-treated blood in the case of chromosome testing for regular congenital abnormalities, and $1.0 \mathrm{ml}$ of bone marrow fluid and $5.0 \mathrm{ml}$ of blood in the case of blood disorders. Currently, no standardized method is available for describing chromosome karyotypes based on SKY analysis.
Table 1 Combination of fluorescent dyes (painting probes) in a chromosome analysis using SKY

\begin{tabular}{|c|c|c|}
\hline SN & 2 & $\mathrm{E}$ \\
\hline & 3 & $\mathrm{ACDE}$ \\
\hline & 4 & $\mathrm{CD}$ \\
\hline & 5 & $\mathrm{ABDE}$ \\
\hline & 6 & $\mathrm{BCDE}$ \\
\hline & 7 & $\mathrm{BC}$ \\
\hline & 8 & $\mathrm{D}$ \\
\hline & 9 & $\mathrm{ADE}$ \\
\hline & 10 & $\mathrm{CE}$ \\
\hline & 11 & $\mathrm{ACD}$ \\
\hline & 12 & $\mathrm{BE}$ \\
\hline & 13 & $\mathrm{AD}$ \\
\hline & 14 & B \\
\hline & 15 & $\mathrm{ABC}$ \\
\hline & 16 & $\mathrm{BD}$ \\
\hline & 17 & $\mathrm{C}$ \\
\hline & 18 & $\mathrm{ABD}$ \\
\hline & 19 & $\mathrm{AC}$ \\
\hline & 20 & A \\
\hline & 21 & $\mathrm{DE}$ \\
\hline & 22 & $\mathrm{ABCE}$ \\
\hline$A$ spectrum orange, $B$ Texas red, & $X$ & $\mathrm{AE}$ \\
\hline $\begin{array}{l}C \text { Cy5, } D \text { spectrum green, } \\
E \text { Cy } 5.5\end{array}$ & $\mathrm{Y}$ & $\mathrm{CDE}$ \\
\hline
\end{tabular}

\section{Characteristics of SKY in Clinical Application}

When a chromosome in which the origin cannot be identified or the structural abnormality of a partial copy of a chromosome is observed with G-banding, the SKY technique makes it possible to recognize each chromosome with different color tones. Furthermore, when cells are cultured under conditions in which the image acquisition of cell division is difficult, SKY enables bleaching and staining of the specimen after the images of division have been verified.

SKY provides the advantage of easy visual interpretation when analyzing results, but it is not possible to evaluate structural abnormalities, such as inversion, deletion, insertion, and duplication in the same chromosome, because these are shown with the same color. Also, neither the Q-positive segment nor the satellite region of the long arm of the $\mathrm{Y}$ chromosome near the centromere can be detected. The resolution limit of detection is approximately 1-2 Mb, similar to conventional chromosome painting techniques, and minor structural abnormalities of less than 1 band cannot be detected. Therefore, in order to verify the site of chromosomal breakage, it is usually necessary to use SKY beforehand in combination with G-banding or 
high-resolution chromosome banding, instead of using SKY alone to macroscopically observe the banding patterns.

Furthermore, SKY provides the following notable advantages. Conventionally, when a chromosome of unknown origin is noticed in a child suffering with some abnormality, a chromosome analysis is performed on both parents in order to make a definitive diagnosis of the chromosome in question. If a balanced structural abnormality is detected in the chromosome of either of the parents, then it is definitively diagnosed as the origin of such a structural abnormality. Conventionally, macroscopic evaluation would be performed based on the banding patterns, in case the informed consent for participation in the karyotype test cannot be obtained from the parents, and in the case of de novo translocation. In such cases, SKY has the advantage of being capable of readily clarifying the chromosome of unknown origin without performing karyotyping of the parents, and also can reveal if one of the parents is a carrier of a balanced structural abnormality. This advantage of SKY is very useful in the clinical practice of genetic counseling [9]. Refer to Table 2 for the indications of evaluation using SKY when structural chromosomal abnormalities are actually found via G-banding.

\section{Case and Review}

In this section, two cases in which SKY can be used are presented, and the benefits and limitations of this technique are discussed. We employed the application of 24-color SKY probes [Applied Spectral Imaging (ASI), Inc., ASI, Carlsbad, CA], skypaint ${ }^{\mathrm{TM}}$ hybridization and detection protocol, SRL Inc., Tokyo, Japan.
Case 1: Trisomy 11/22

The results of chromosome analysis on Trisomy 11/22 using SKY are shown in Fig. $1[8,9]$. SKY was performed after an extra marker chromosome was detected in group $\mathrm{G}$ chromosomes via G-banding and the results showed the trisomy of chromosome 22. One of the extra chromosomes was distinguished by two colors, and it was deduced to be a derivative chromosome [der(22)], likely resulting from the reciprocal translocation in which the long arms of chromosome 11 (q23.3) and chromosome 22 (q11.2) were the breakpoints. The karyotype of this case is described as $+\operatorname{der}(22) \mathrm{t}(11: 22)(\mathrm{q} 23.3 ; \mathrm{q} 11.2)$. Therefore, SKY is one of the most effective approaches at present for the identification of an extra chromosome of unknown origin. With the use of SKY technique, it was possible to make a definitive diagnosis of trisomy $11 / 22$ in the child without performing any chromosome testing on the parents, i.e., without examining the possibility of structural abnormalities that would have been inherited from either one of the parents. This is significant in the clinical practice of genetic counseling for parents to maintain their relationship.

\section{Case 2: 13q-Syndrome}

The results of the chromosome analysis of 13q-syndrome using SKY (Fig. 2) indicate structural abnormalities in chromosomes 2, 4, and 13 [12, 21]. For chromosomes 2 and 4 , the reciprocal translocation $\mathrm{t}(2 ; 4)$ with breakpoints on the long arm of chromosome 2 (q24.2) and the short arm of chromosome 4 (p14) can be readily distinguished. This $\mathrm{t}(2 ; 4)$, however, may involve [inv(4)(p15.1p15.32)], which is a paracentric inversion with breakpoints at p15.1 and

Table 2 Usability when analyzing abnormalities that have been found via G-banding by using SKY

\begin{tabular}{|c|c|c|c|}
\hline Result of G-banding & Karyotype & Supplementary note on SKY & Usability of SKY \\
\hline Accessory chromosome & add & Identifies the derivative chromosome of an extra portion & a \\
\hline Derivative chromosome & der & Identifies the related chromosomes & a \\
\hline Insertion & ins & Identifies the derivative chromosome of insertion fragment & a \\
\hline A complicated translocation & & Identifies the related chromosomes & a \\
\hline Genetic amplification & $\operatorname{dimin} / \mathrm{hsr}$ & Identifies the derivative chromosome of homogeneous staining region & a \\
\hline Duplication? & ?dup & Identifies any duplication & a \\
\hline Reciprocal translocation & $\mathrm{t}$ & Same analysis level of G-banding & $\mathrm{b}$ \\
\hline Inversion & inv & Difficult to make an analysis that is better than G-banding & c \\
\hline Deletion & del & Difficult to make an analysis that is better than G-banding & c \\
\hline Numerical aberrations & $+/-$ & Difficult to make an analysis that is better than G-banding & c \\
\hline Unknown of derivative & mar & Identifies a derivative chromosome within the limitations of sensitivity & c \\
\hline Cryptic translocation & & Detects a derivative chromosome within the limitations of sensitivity & c \\
\hline
\end{tabular}




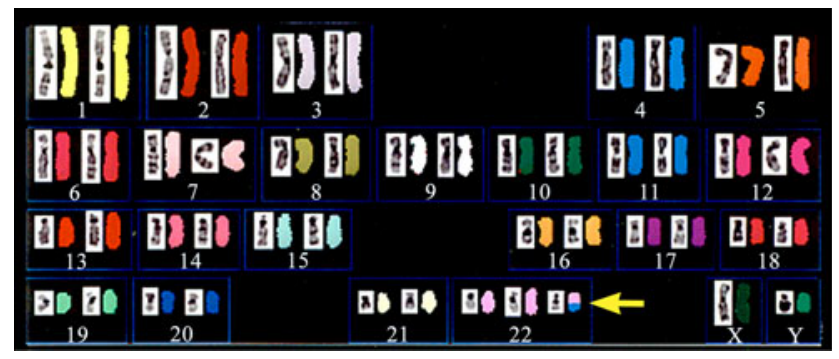

Fig. 1 Chromosome analysis on Trisomy 11/22 using SKY

p15.32 in the short arm part of chromosome 4 that has been translocated to chromosome 2 , and this necessitates a verification via high-resolution chromosome banding. Inversions occurring in the same chromosome are not distinguished with color, so it is impossible to make a definitive diagnosis by using SKY. Thus in this case, testing of the parents' chromosomes via high-resolution chromosome banding is necessary for further detailed diagnosis.

For chromosome 13, it is possible to diagnose the occurrence of a deletion that is identified as $13 \mathrm{q}$ minus syndrome by using SKY only, but this does not reveal information about the breakpoints. By simultaneously combining this SKY-derived information with the banding patterns to be tested, it can be diagnosed as a paracentric deletion with breakpoints at q21.2 and q31.2. The karyotype of Case 2 is described as 46,XX, der(2)t(2:4)(q24.2;p14)inv(4) (p15. $1 \mathrm{p} 15.32), \operatorname{der}(4) \mathrm{t}(2 ; 4), \operatorname{del}(13)(\mathrm{q} 21.2 \mathrm{q} 31.2)$ or $46, \mathrm{XX}, \mathrm{t}(2 ; 4)$ (q24.2;p14), del(13)(q21.2q31.2). As stated above, it is difficult to make a definitive diagnosis from the results of a SKY analysis alone, and also one needs to be careful, because the conventional method of G-band staining cannot be used directly as a stand-alone substitute. Thus, more reliable interpretation and diagnosis can be made by combining the information from the two separate techniques, as neither of them can be used for unequivocal diagnosis.

When performing tests using SKY, it is necessary to understand the characteristics and the limitations of this analysis, and to combine the test results with those of

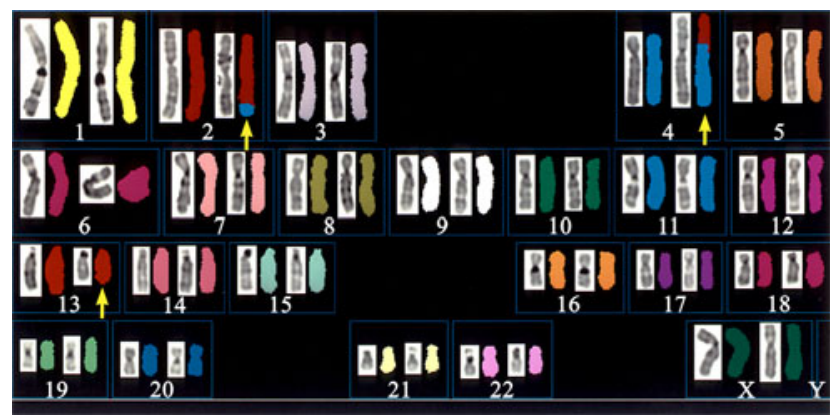

Fig. 2 Chromosome analysis of 13q-syndrome using SKY showing structural abnormalities in chromosomes 2,4 , and 13
G-band staining or high-resolution chromosome banding for a comprehensive karyotypic diagnosis.

\section{Conclusion}

With the introduction of SKY, a new technology of chromosome analysis, the accuracy of testing is improved and it now becomes possible to devise new clinical studies that previously had not been feasible with conventional testing methods. T-Band regions such as 1p, 11p15, 16p, 19p, 19q, and $22 \mathrm{q}$ are known as sites that are difficult to observe as bands of contrasting colors, even though it is believed that these regions contain many genes, according to conventional chromosome analysis. Furthermore, the macroscopic analysis of that band with the human eye involves a limitation in recognizing minor reciprocal translocations, and it is also possible that they may go unnoticed. Identification via SKY coloring has overcome the weakness of the conventional band pattern diagnosis method, and it is expected that other previously unrecognized specific chromosomal translocations are likely to be revealed with the widespread use of this technique.

Moreover, in recent years, spectral color banding has been developed as a technique that has overcome the limitations of SKY [22] and in this technique G-band differential staining is combined with the SKY coloring technology. Clinical use of this novel technique is expected to be widespread in the field of clinical genetics in the future and to make significant contributions. However, the cost of this technique still remains prohibitory. Considering these points, from the standpoint of genetic counseling, incorporation of SKY into G-band chromosome banding or high-resolution chromosome banding as needed would be the most practical technique of chromosome testing. In fact, when the structural abnormality of a chromosome of unknown origin is detected via G-banding, the combined use of SKY makes it possible to identify the origin without performing karyotyping of the parents, which could identify which parent is the carrier of the balanced structural abnormality. Therefore, in the clinical practice of genetic counseling, informed consent for a test of congenital chromosomal abnormalities can be readily obtained from the parents. Furthermore, an evaluation of the SKY results is made based on the colors that are displayed, so it is easy for a physician to explain the results to the parents of a patient. This advantage could potentially reduce the unnecessary psychological burden on parents with a child who demonstrates a structural abnormality in a chromosome.

Acknowledgments The authors would like to thank SRL Inc., Tokyo, Japan for executing cytogenic analysis of SKY and FISH technique. This study was supported by Dokkyo Medical University Investigator-Investigated Research Grant (No. 2010-01-6). 
Open Access This article is distributed under the terms of the Creative Commons Attribution Noncommercial License which permits any noncommercial use, distribution, and reproduction in any medium, provided the original author(s) and source are credited.

\section{References}

1. Harper, P. S. (2006). The discovery of the human chromosome number in Lund, 1955-1956. Human Genetics, 119, 226-232.

2. Lejeune, J., Turpin, R., \& Gautier, M. (1959). Mongolism; a chromosomal disease (trisomy). Bulletin de l'Academie Nationale de Medecine, 143, 256-265.

3. Imataka, G., Mitsui, M., Mitsui, N., Hirabayashi, H., Yamanouchi, H., et al. (2005). Down syndrome with acute epiglottitis. Pediatrics International, 47, 333-335.

4. Edwards, J. H., Harnden, D. G., Cameron, A. H., Crosse, V. M., \& Wolff, O. H. (1960). A new trisomic syndrome. Lancet, 1, 787-790.

5. Imataka, G., Nitta, A., Suzumura, H., Watanabe, H., Yamanouchi, H., et al. (2007). Survival of trisomy 18 cases in Japan. Genetic Counseling, 18, 303-308.

6. Patau, K., Smith, D. W., Therman, E., Inhorn, S. L., \& Wagner, H. P. (1960). Multiple congenital anomaly caused by an extra autosome. Lancet, 1, 790-793.

7. de Grouchy, J., Turleau, C., \& Leonard, C. (1971). Study by fluorescence of a trisomy C mosaic, probably 8: 46, XY-47, XY,?8+. Annales de Genetique, 14, 69-72.

8. Iselius, L., Lindsten, J., Aurias, A., Fraccaro, M., Bastard, C., et al. (1983). The 11q;22q translocation: A collaborative study of 20 new cases and analysis of 110 families. Human Genetics, 64, 343-355.

9. Imataka, G., Takaya, Y., Hagisawa, S., Yamanouchi, H., \& Eguchi, M. (2004). Trisomy 11/22 diagnosed by spectral karyotyping (SKY). Genetic Counseling, 15, 391-394.

10. Ford, C. E., Jones, K. W., Polani, P. E., De Almeida, J. C., \& Briggs, J. H. (1959). A sex-chromosome anomaly in a case of gonadal dysgenesis (Turner's syndrome). Lancet, 1, 711-713.
11. Tanner, J. M., Prader, A., Habich, H., \& Ferguson-Smith, M. A. (1959). Genes on the Y chromosome influencing rate of maturation in man: Skeletal age studies in children with Klinefelter's (XXY) and Turner's (XO) syndromes. Lancet, 2, 141-144.

12. Yamanouchi, H., Imataka, G., Nakagawa, E., Nitta, A., Suzuki, N., et al. (2005). An analysis of epilepsy with chromosomal abnormalities. Brain and Development, 27, 370-377.

13. Moorhead, P. S., Nowell, P. C., Mellman, W. J., Battips, D. M., \& Hungerford, D. A. (1960). Chromosome preparations of leukocytes cultured from human peripheral blood. Experimental Cell Research, 20, 613-616.

14. Nowell, P. C., \& Hungerford, D. A. (1960). Chromosome studies on normal and leukemic human leukocytes. Journal of the National Cancer Institute, 25, 85-109.

15. Watson, J. D. (1990). The human genome project: Past, present, and future. Science, 248, 44-49.

16. Caspersson, T., Castleman, K. R., Lomakka, G., Modest, E. J., Moller, A., et al. (1971). Automatic karyotyping of quinacrine mustard stained human chromosomes. Experimental Cell Research, 67, 233-235.

17. Yunis, J. J., \& Chandler, M. E. (1978). High-resolution chromosome analysis in clinical medicine. Progress in Clinical Pathology, 7, 267-288.

18. Trask, B. J. (1991). Fluorescence in situ hybridization: Applications in cytogenetics and gene mapping. Trends in Genetics, 7, $149-154$.

19. Kallioniemi, A., Kallioniemi, O. P., Sudar, D., Rutovitz, D., Gray, J. W., et al. (1992). Comparative genomic hybridization for molecular cytogenetic analysis of solid tumors. Science, 258, 818-821.

20. Schrock, E., du Manoir, S., Veldman, T., Schoell, B., Wienberg, J., et al. (1996). Multicolor spectral karyotyping of human chromosomes. Science, 273, 494-497.

21. Allderdice, P. W., Davis, J. G., Miller, O. J., Klinger, H. P., Warburton, D., et al. (1969). The 13q-deletion syndrome. American Journal of Human Genetics, 21, 499-512.

22. Kakazu, N., Ashihara, E., Hada, S., Ueda, T., Sasaki, H., et al. (2001). Development of spectral colour banding in cytogenetic analysis. Lancet, 357, 529-530. 